\title{
Long term results of mechanical prostheses for treatment of active infective endocarditis
}

\author{
J M Guerra, M P Tornos, G Permanyer-Miralda, B Almirante, M Murtra, J Soler-Soler
}

\begin{abstract}
Objective-To analyse the long term results of mechanical prostheses for treating active infective endocarditis.

Design-Prospective cohort study of a consecutive series of patients diagnosed with infective endocarditis and operated on in the active phase of the infection for insertion of a mechanical prosthesis.

Setting-Tertiary referral centre in a metropolitan area.

Results-Between 1975 and 1997, 637 cases of infective endocarditis were diagnosed in the centre. Of these, 436 were left sided (with overall mortality of $20.3 \%$ ). Surgical treatment in the active phase of the infection was needed in 141 patients ( $72 \%$ native, $28 \%$ prosthetic infective endocarditis). Mechanical prostheses were used in 131 patients. Operative mortality was 30.5\% (40 patients). Ninety one survivors were followed up prospectively for (mean (SD)) 5.4 (4.5) years. Thirteen patients developed prosthetic valve dysfunction. Nine patients suffered reinfection: four of these $(4 \%)$ were early and five were late. The median time from surgery for late reinfection was 1.4 years. During follow up, 12 patients died. Excluding operative mortality, actuarial survival was $86.6 \%$ at five years and $83.7 \%$ at 10 years; actuarial survival free from death, reoperation, and reinfection was $73.1 \%$ at five years and $59.8 \%$ at 10 years.

Conclusions-In patients surviving acute infective endocarditis and receiving mechanical prostheses, the rate of early reinfection compares well with reported results of homografts. In addition, prosthesis dysfunction rate is low and long term survival is good. These data should prove useful for comparison with long term studies, when available, using other types of valve surgery in active infective endocarditis.
\end{abstract}

(Heart 2001;86:63-68)

Keywords: infective endocarditis; surgery; mechanical prosthesis

The diagnosis and treatment of infective endocarditis has evolved greatly in recent years. Nevertheless, the morbidity and mortality of this disease remains high. ${ }^{1-4}$ Initially, medical treatment with an effective antibiotic is mandatory. If the disease progresses or heart failure symptoms develop because of valve disruption, surgery should be considered. ${ }^{5-9}$ Thus the surgical treatment must often be performed in the active phase of the infection. The best timing for the operation and the preferred surgical technique are matters of debate. The most controversial points are the optimal device for the valve replacement and the long term results of such surgery.

Our aim in this study was to analyse the long term results of mechanical prostheses for the treatment of active infective endocarditis in our institution during a 22 year period. We report a large prospective series with standardised preoperative management, surgical indications, and long term follow up.

Vall d'Hent, Hospital d'Hebron 119-129, 08035 Barcelona, Spain J M Guerra

M P Tornos

G Permanyer-Miralda

B Almirante

M Murtra

J Soler-Soler

Correspondence to:

Dr Guerra

jmguerra@hg.vhebron.es

Accepted 31 January 2001 out the patients' management jointly. During the period of study, 637 cases of infective endocarditis were diagnosed. Of those, 436 $(68.4 \%)$ were left sided, $314(71.8 \%)$ were native infective endocarditis, and $122(28.2 \%)$ were prosthetic infective endocarditis. The overall mortality of left sided infective endocarditis was $20.3 \%$. Surgical treatment in the active phase of the illness was needed in 141 patients $(32.3 \%)$. A mechanical prosthesis was implanted in 131 patients, and these constitute the study group. The nine patients who received a biological prosthesis and one with a mitral valve repair were excluded from the study.

\section{DEFINITIONS}

The diagnosis of infective endocarditis was made according to criteria proposed by Durack and colleagues, ${ }^{10}$ and these were retrospectively applied to cases diagnosed before their publication. All patients included in the present analysis fulfilled the criteria of definite infective endocarditis. Culture negative endocarditis was present when no microorganism could be identified on serial blood cultures, in the material extracted from the surgical intervention, or in serological studies.

Infective endocarditis was considered active if the patient required surgery before completion of a standard course of antibiotic treatment, irrespective of whether there were ongoing signs of sepsis or whether the blood 
cultures, surgical specimens, or both were positive for the infecting organism. Prosthetic valve endocarditis was defined as infection occurring on any type of tissue or mechanical valve device. Early prosthetic valve endocarditis was present if infective endocarditis occurred within a year after surgery. Endocarditis occurring after a year was defined as late prosthetic valve endocarditis.

Emergency surgery was defined as surgery for patients with life threatening conditions that could not be postponed for more than 24 hours, and elective surgery comprised those operations that could be delayed for a few days without risk to the patient. The infection was considered uncontrolled at the time of operation when positive blood cultures were obtained within 48 hours before surgery, or when clinical manifestations of sepsis were apparent at that time. Operative mortality was defined as death occurring within 30 days after surgery or during the same hospital stay.

\section{TREATMENT}

Antibiotic treatment was based on the established guidelines of the cardiology and infectious diseases departments at our institution. All patients received antimicrobial treatment according to antimicrobial susceptibility. Culture negative endocarditis was treated with combined broad spectrum antibiotics.

The indications for surgery were based on our established guidelines. The indications for surgery in the active phase of the illness are:

- presence of heart failure secondary to valvar regurgitation;

- signs suggesting myocardial invasion (atrioventricular block, echocardiographic features of abscesses);

- persistence of sepsis after a week of appropriate antibiotic treatment;

- infective endocarditis from brucella or fungi;

- repetitive emboli;

- early prosthetic endocarditis;

- late prosthetic endocarditis from Staphylococcus aureus;

- prosthetic valve dysfunction.

In all patients, the surgical operation included radical excision of the infected tissue, closure of cavities and fistulae, resection of the infected valve, and its replacement by a mechanical prosthesis.

FOLLOW UP

Three serial blood cultures and an echocardiogram were carried out in all patients after the end of antibiotic treatment, before discharge. Three additional blood cultures were performed after one month. After discharge, the same group of doctors followed all patients, initially every six months and later on annually. Each visit included a physical examination and an echocardiogram. The patients were instructed to present to the hospital immediately if fever or symptoms of heart failure arose.

DATA SAMPLING AND STATISTICAL METHODS Data regarding hospital admissions and clinical status on follow up were obtained from the hospital records. All surgical registers were reviewed. Occasionally, patients or their relatives were contacted to assess a particular event. Complete follow up was available for $90.1 \%$ of the patients. The follow up was partial for the remaining $9.9 \%$, as patients were lost after some follow up visits. Loss occurred in all cases after the first year (mean (SD), 5.5 (3.5) years, range 1.1 to 12.0 years), mainly because of change of address. Some follow up data were thus available in all patients.

\section{STATISTICS}

Data are given as mean (SD) or median (range) where appropriate. Bivariate analysis of the differences between groups was done with the $\chi^{2}$ or Fisher's exact test for proportional variables and with Student's $t$ test for continuous variables. Multivariate stepwise logistic regression analysis was used to identify independent variables predicting operative mortality and events during follow up. Cumulative survival and event-free periods were estimated by the Kaplan-Meier method. Differences in survival at follow up were estimated by use of log-rank tests. The statistical analysis was performed with the SPSS statistical package (SPSS Inc, Chicago, Illinois, USA). The level of significance was set at $\mathrm{p} \leqslant 0.05$.

\section{Results}

PATIENTS

During the study period, 131 patients were operated on (98 male $(74.8 \%)$ and 33 female $(25.2 \%))$. Their mean (SD) age was 51 (14) years, range 15 to 77 . A native infective endocarditis was present in 94 cases $(71.8 \%)$ and a prosthetic infective endocarditis in $37(28.2 \%)$, 19 of which were early and 18 late infections. Of the cases of prosthetic infective endocarditis, the infection affected a mechanical prosthesis in 33 cases and a biological prosthesis in four.

General characteristics of the population are presented in table 1 and the results of the microbiological investigations in table 2 .

PREOPERATIVE ASSESSMENT AND TREATMENT Preoperative serial blood cultures were positive in 101 cases $(77.1 \%)$. The diagnosis of infective endocarditis caused by Coxiella burnetii was based on serological studies in four cases. As a result of the microbiological studies performed on surgical specimens, an aetiological diagnosis could be made in 11 patients with negative blood cultures. Thus an infecting microorganism could be established in 109 patients $(83.2 \%)$.

Forty patients $(30.5 \%)$ suffered embolic phenomena: $18(13.7 \%)$ ischaemic strokes, five $(3.8 \%)$ coronary embolisms, and 14 $(10.7 \%)$ multiple embolisms. Four patients (3.1\%) developed complete atrioventricular block.

Median duration of antibiotic treatment in the whole population before surgery was nine days (range 0-90 days). Indications for surgery in patients with native valve infective endocarditis were: progressive heart failure in 75 $(79.8 \%)$, repetitive emboli in three $(3.2 \%)$, 


\section{Table 1 Patient characteristics}

\begin{tabular}{ll}
\hline Variable & \\
\hline Number of patients & 131 \\
Sex & \\
$\quad$ Male & $98(74.8 \%)$ \\
Female & $33(25.2 \%)$ \\
Age (years) & $53(15$ to 77$)$ \\
Native valve infective endocarditis & $94(71.8 \%)$ \\
Prosthetic valve infective endocarditis & \\
Early & $19(14.5 \%)$ \\
Late & $18(13.7 \%)$ \\
Location & \\
Aortic & $67(51.2 \%)$ \\
Mitral & $32(24.4 \%)$ \\
Aortic and mitral & $32(24.4 \%)$ \\
Duration of symptoms before diagnosis (days) & $21(0$ to 365$)$ \\
Diabetes mellitus & $23(17.6 \%)$ \\
Chronic obstructive pulmonary disease & $16(12.2 \%)$ \\
History of ischaemic heart disease & $6(4.6 \%)$ \\
Chronic renal failure & \\
$\quad$ On haemodialysis & $2(1.5 \%)$ \\
$\quad$ Not on haemodialysis & $8(6.1 \%)$ \\
Preoperative coronary arteriography & $35(26.7 \%)$ \\
Duration of antibiotic treatment before operation (days) & $9(0$ to 90$)$ \\
Uncontrolled infection at time of intervention & $26(19.8 \%)$ \\
NYHA functional class at the time of operation & \\
I-II & $12(9.2 \%)$ \\
III-IV & $105(80.1 \%)$ \\
Cardiogenic shock & $14(10.7 \%)$ \\
Emergency surgery & $16(12.2 \%)$ \\
Duration of antibiotic treatment after operation (days) & $22(0$ to 1125$)$ \\
\hline Valus are (\%) or median (range) & \\
\hline
\end{tabular}

Values are $\mathrm{n}(\%)$ or median (range).

Table 2 Microorganisms causing infective endocarditis in 131 patients

\begin{tabular}{lrr}
\hline Organism & $n$ & $\%$ \\
\hline Staphylococci & 19 & 14.5 \\
$\quad$ Coagulase positive & 19 & 14.5 \\
$\quad$ Coagulase negative & 18 & 13.7 \\
Streptococci & 6 & 4.6 \\
$\quad$ Viridans group & 7 & 5.4 \\
Streptococcus bovis & 7 & 5.4 \\
$\quad$ Haemolytic & 13 & 9.9 \\
S pneumoniae & 4 & 3.0 \\
Enterococcus faecalis & 16 & 12.2 \\
HACEK group & 22 & 16.8 \\
Miscellaneous & \\
Culture negative & \\
\hline
\end{tabular}

HACEK: haemophilus, actinobacillus, cardiobacterium, eikenella, kingella.

${ }^{\star}$ Coxiella burnetii (4), Pseudomona aeruginosa (3), Brucella spp (2), Corynebacterium spp (2), Candida parapsilosis (1), Listeria monocytogenes (1), Neisseria spp (1), Peptococcus spp (1), Capnocytophaga canimusus (1).

virulent microorganism in three $(3.2 \%)$, atrioventricular block in one $(1.1 \%)$, and a combination of indications in $12(12.8 \%)$. Indications for surgery for patients with late prosthetic infective endocarditis were: progressive heart failure with prosthesis dysfunction in $14(77.8 \%)$, infective endocarditis caused by Staphylococcus aureus in two (11.1\%), and prosthetic valve dysfunction without heart failure in two $(11.1 \%)$.

SURGICAL TREATMENT AND POSTOPERATIVE CARE Surgery was indicated as an emergency because of clinical instability or haemodynamic impairment in 16 patients $(12.2 \%)$. Elective surgery was performed in the remaining 115 patients, with a median delay between diagnosis and surgery of 12 days.

Five patients simultaneously underwent revascularisation surgery and in four a permanent pacemaker was implanted. Two patients with hypertrophic cardiomyopathy each underwent a Morrow's myectomy at the same time.

The median duration of postoperative antibiotic treatment was 22 days (range 0-1125 days). Fifty eight patients developed deterioration of serum creatinine concentrations postoperatively, requiring dialysis in 14 (10.7\%).

OPERATIVE MORTALITY

Operative mortality was $30.5 \%$ (40 patients). The causes of death were cardiogenic shock in 14 patients, intraoperative death in seven, septic shock in five, prosthesis dysfunction in three, haemorrhage in three, multiorgan failure in two, cardiorespiratory arrest of unknown cause in two, arrhythmias in one, intestinal necrosis in one, and anoxic coma in one. Bivariate analysis showed significant associations with operative mortality for increased age (56 v 49 years), female sex (40\% v 19\%), shorter duration of symptoms before diagnosis (28 $v 45$ days), longer duration of cardiopulmonary bypass and longer duration of ischaemia at operation (112 v82 minutes and $71 v 58$ minutes, respectively), and the involvement of both left valves (37\% v 18\%). Emergency surgery $(20 \% v 9 \%)$, advanced New York Heart Association (NYHA) functional class before operation ( $80 \%$ class III-IV and $17 \%$ in cardiogenic shock $v 80 \%$ class III-IV and $8 \%$ in cardiogenic shock), and the presence of abscesses at the operation $(37 \%$ v $23 \%)$ showed a non-significant trend towards increased operative mortality. Multivariate analysis showed significant associations only for increased age, shorter duration of symptoms before diagnosis, emergency surgery, and longer time of bypass at operation (table 3). A large proportion of the patients in the sample population had one or more of these risk factors (for example, 18\% were more than 65 years old, $25 \%$ were female, $24 \%$ had involvement of both left valves, $91 \%$ were in advanced NYHA functional class before operation, and $27 \%$ had abscesses at operation). The time when the operations were carried out (before or after 1985) was not associated with any significant difference in the clinical results.

FOLLOW UP

Ninety one patients survived. All patients were prospectively followed up for a mean period of 5.4 (4.5) years (range 26 days to 18.9 years). Twelve patients died during follow up: five from cardiac causes (sudden death in three, heart failure in one, new infective endocarditis in one), five from non-cardiac cause (malignancy in two, stroke in one, respiratory failure in one, vascular disease in one), and in two patients the cause of death could not be determined. Actuarial survival at five and 10 years for all the patients, including operative mortality, was 60.1 (4.5)\% and 58.1 (4.8)\%, respectively (fig 1). Actuarial survival at five and 10 years for patients surviving the surgery was $86.6(4.0) \%$ and 83.7 (4.8)\%. Multivariate analysis showed significant differences between groups for the presence of chronic obstructive pulmonary disease and longer length of stay in the postoperative care unit. No differences in survival were detected between native and prosthetic infective endocarditis.

Thirteen patients developed prosthetic valve dysfunction, in four cases occurring in the early 
Table 3 Bivariate and multivariate analysis of risk factors influencing operative mortality

\begin{tabular}{|c|c|c|c|}
\hline \multirow[b]{2}{*}{ Patient risk characteristics } & \multicolumn{2}{|l|}{$p$ Value } & \multirow{2}{*}{$\begin{array}{l}\text { Multivariate analysis } \\
\text { OR }(95 \% C I)\end{array}$} \\
\hline & $B V$ & $M V$ & \\
\hline Increased age & 0.012 & 0.029 & $1.05(1.01$ to 1.10$)$ \\
\hline Female sex & 0.015 & - & \\
\hline Prosthetic valve infective endocarditis & 0.53 & - & \\
\hline Aortic valve infective endocarditis & 0.83 & - & \\
\hline Multivalve involvement & 0.028 & - & \\
\hline Diabetes mellitus & 0.62 & - & \\
\hline Shorter duration of symptoms before diagnosis & 0.050 & 0.026 & $1.03(1.01$ to 1.04$)$ \\
\hline Systemic emboli & 0.84 & - & \\
\hline Increased preoperative creatinine levels & 0.15 & - & \\
\hline Chronic renal failure & 1.00 & - & \\
\hline Atrioventricular block & 1.00 & - & \\
\hline $\begin{array}{l}\text { Shorter duration of antibiotic treatment before } \\
\text { operation }\end{array}$ & 0.51 & - & \\
\hline Uncontrolled infection at intervention & 0.64 & - & \\
\hline Endocarditis caused by staphylococci & 0.40 & - & \\
\hline Culture negative endocarditis & 0.80 & - & \\
\hline Advanced NYHA functional class & 0.07 & - & \\
\hline Preoperative shock & 0.125 & - & \\
\hline Emergency surgery & 0.086 & 0.003 & $12.5(2.28$ to 60.24$)$ \\
\hline Surgery before 1985 & 0.52 & - & \\
\hline Presence of abscesses at operation & 0.095 & - & \\
\hline Longer duration of ischaemia at operation & 0.004 & - & \\
\hline Longer time of bypass at operation & $<0.001$ & 0.0001 & $1.04(1.02$ to 1.06$)$ \\
\hline
\end{tabular}

$\mathrm{BV}$, bivariate; $\mathrm{MV}$, multivariate; $\mathrm{OR}$, odds ratio; $\mathrm{CI}$, confidence interval.

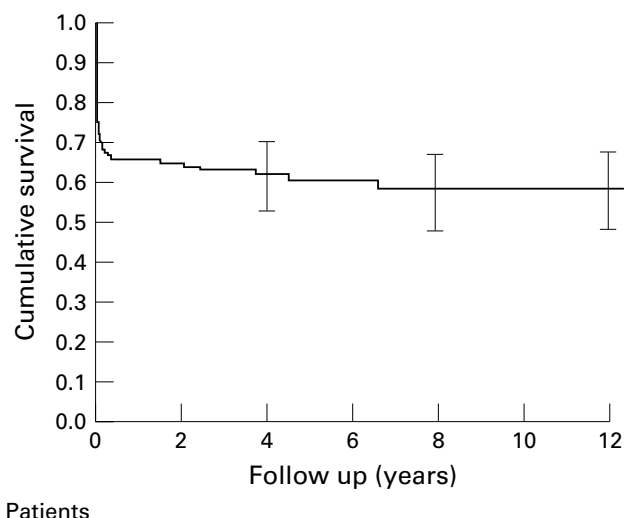

$\begin{array}{lllllll}\text { at risk: } 131 & 67 & 51 & 30 & 22 & 16 & 8\end{array}$

Figure 1 Actuarial survival curve for patients operated on for active infective endocarditis using a mechanical prosthesis. Bars represent the 95\% confidence interval for each period.

postoperative period (during admission): two were operated on (one survived the operation and the other died intraoperatively) and two died before the operation could be performed. The other nine patients developed prosthetic valve dysfunction during the follow up period, leading to valve replacement with a mechanical prosthesis in all but one. Eight had a periprosthetic leak and one presented with a prosthetic valve thrombosis which was surgically treated. None of these died at reoperation. Mean time until detection of the prosthesis dysfunction in the group of patients who were initially discharged was 2.2 (2.0) years (range 210 days to 5.6 years). Younger age, atrioventricular block, presence of abscesses, and early year of surgery were significant in bivariate analysis. Multivariate analysis showed significance for the presence of atrioventricular block and earlier period of surgery.

Reinfection occurred in nine patients $(6.9 \%$ of survivors). Four patients developed early infective endocarditis at 38,63,111, and 295 days, respectively. Three of these were considered to be recurrent infections, as the causal

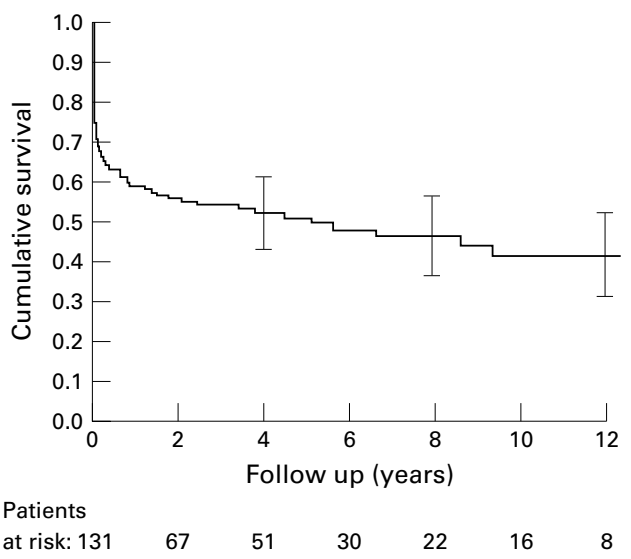

Figure 2 Actuarial survival curve free from death, reoperation, or reinfection for patients operated on for active infective endocarditis using a mechanical prosthesis. Bars represent the $95 \%$ confidence interval for each period.

microorganisms were different from those initially isolated. Pseudomonas species could be identified in both infections in the patient developing further clinical infective endocarditis at 63 days, and this case was thus considered a relapse. Eight patients were operated on. The patient with the relapse died before an operation could be performed. Actuarial survival free from reinfection in the patients surviving surgery at five and 10 years was 92.8 (2.8)\% and $83.0(7.0) \%$, respectively. No variables showed significance on bivariate or multivariate analysis.

Considering all types of events together, actuarial survival free from death, reinfection, or reoperation in the whole population at five and 10 years was 50.8 (4.5)\% and $41.6(5.4) \%$ (fig 2). If operative mortality is excluded, actuarial survival at five and 10 years was 73.2 (4.8)\% and 59.8 (6.9)\%, respectively.

\section{Discussion}

The best therapeutic algorithm for infective endocarditis is still debatable..$^{5-811-19}$ It is clear that broad spectrum antibiotic treatment must be given initially. Progression of the disease or the appearance of signs of heart failure despite adequate treatment makes a surgical approach mandatory. Thus surgery is often undertaken in the active phase of the disease. Likewise, when spread of the infection or disruption of the valve leaflets does not allow conservative management to continue, it is often necessary to perform a valve replacement. Nevertheless, optimal results are not achieved. ${ }^{13561720-23}$ Some reports have suggested that the use of a homograft allows better short and medium term results. ${ }^{17} 1822{ }^{24-29}$ It therefore seems very important to show whether the use of homografts, which is difficult and requires highly specialised equipment, could improve the results obtained with mechanical prostheses, especially as a randomised study comparing the two approaches is hardly feasible.

We present here our experience over 22 years in the management of infective endocarditis, limited to the patients who underwent valve replacement with a mechanical prosthesis during the active phase of the disease. All the 
patients treated for infective endocarditis were considered for inclusion in the series. Most were primary diagnoses in our institution and the rest were referred to us in our role as a tertiary referral centre. The sample is therefore probably not representative of the population as a whole, as the patients referred from other centres represent a more severely affected subgroup at increased risk. With respect to the study design and aims, this bias is likely to assume less importance when evaluating long term postoperative results.

Our centre's cardiology and infectious diseases departments jointly managed all the patients. In addition, indications for surgery were strictly based on clinical guidelines approved in our institution. This enhanced our ability to interpret the results. The medical staff policy was not to reject any patient for surgery because of age, coexisting disease, or a compromised clinical or haemodynamic situation. We therefore believe that all patients suffering from infective endocarditis - and hence all potential candidates for surgery-were included in the study. This explains the relatively high operative mortality in our series. However, it is difficult to compare our results with those of other studies in which the population characteristics are not clearly stated. We cannot exclude the possibility that in some published reports the selection of patients in better condition before surgical intervention resulted in a lower rate of complications and as a result in better short and medium term results.

As previously stated, our operative mortality was high. However, patients operated on in these circumstances have severe disease, with a high prevalence of factors associated with surgical death in bivariate and multivariate analysis and a global mortality as high as $20 \%$ even in the absence of surgery. Risk factor analysis for operative mortality has shown results comparable to those of other studies, particularly in unselected populations. ${ }^{321}$ One could speculate that the use of homografts might have reduced our operative mortality, by lessening early postoperative valve dysfunction and avoiding some intraoperative deaths. We cannot definitely exclude this hypothesis, although the limitations of studies with homografts are discussed below. In addition, the aim of our study was not to demonstrate the superiority or otherwise of mechanical valves, but to show the results of using these devices, which are more widely available than homografts in all institutions. It must be admitted that our mortality rates have not been reduced in recent years with improvements in surgical techniques and postoperative care. This may reflect the importance of the baseline characteristics of the population. Our group has in fact addressed the issue of the persistence of a high mortality in infective endocarditis in relation to changes in the population, the microorganisms involved, and the clinical features of the disease over the years. ${ }^{30}$

All our patients were prospectively followed up in the outpatient clinic after discharge, and were mainly managed by the same medical team. This enhances the accuracy of the prospectively collected data.

In patients surviving surgery, late mortality - as shown in fig 1 -was good $(16 \%$ at 10 years). The rate of reinfection or dysfunction in the short and long term compares quite well with that of previously reported studies. ${ }^{3212231}$ However, with a reoperation rate of $20 \%$ in the survivors, the management of the condition remains far from optimal. Many investigators support the use of homografts for reducing complication rates. ${ }^{17} 182224-2628$ Although there are studies claiming that homografts reduce the likelihood of reinfection, the global reoperation rate with homografts is similar to that with mechanical prostheses, ${ }^{22} 32$ and one report showed no clear superiority for homografts. ${ }^{33}$ Most of these series have included only small numbers of patients with a short follow up. Selection bias in these series also cannot be excluded, particularly in series that include both patients receiving mechanical prostheses and patients receiving homografts. In practice, the methodological limitations and the heterogeneity of available reports make it impossible to demonstrate the long term superiority of any one type of valve substitute over the others.

These limitations of studies using homografts are common to most reported series on the surgical treatment of infective endocarditis, which are mainly based on the retrospective analysis of the surgical experience of different groups. $^{5} 68912-161819$ 21-29 31-35 The number of patients studied is variable and often small. These studies are usually not randomised and cover long periods of time. Several of them include patients suffering from active infective endocarditis and patients who were operated on when the infection was cured. For these various reasons, observed differences in the results may be attributable not only to the device implanted but also to differences in patient selection, surgical technique, preoperative management, and postoperative care. These variables make data analysis and intergroup comparisons extremely difficult.

\section{CONCLUSIONS}

We believe our long term results are reasonably satisfactory and reflect the current management of infective endocarditis. The short term results can also be considered acceptable, as the high operative mortality appears to be associated with our particularly high risk population and is to be expected with the use of other types of device for heart valve replacement.

Taking into account the advantages of using mechanical prostheses because of their widespread availability, and the limitations of existing studies on other valve devices, we support the general use of mechanical prostheses for treating active infective endocarditis when valve repair is not possible. If homografts are available they should be used in circumstances where there is severe disruption of anatomical structures or technical problems in implanting mechanical devices. Our data should prove 
useful for comparisons with other series, when available, using other types of valve substitute.

1 Acar J, Michel PL, Varenne O, et al. Surgical treatment of infective endocarditis. Eur Heart f 1995;16(suppl B):94-8.

infective endocarditis. Eur Heart f 1995;16(suppl B): $94-8$. clinical spectrum and prognosis of native valve infective clinical spectrum and prognosis of native valve infective
endocarditis in non-addicts changing? Eur Heart $\mathcal{f}$ endocarditis in

3 Verheul HA, Van den Brink RB, Van Vreeland T, et al. Effects of changes in management of active infective endocarditis on outcome in a 25 -year period. Am $\mathcal{f}$ Cardiol 1993;72:682-7.

4 Castillo JC, Anguita MP, Ramírez A, et al. Long term outcome of infective endocarditis in patients who were not drug addicts: a 10 year study. Heart 2000;83:525-30.

5 Dodge A, Hurni M, Ruchat P, et al. Surgery in native valve endocarditis: indications, results and risk factors. Eur $\mathcal{F}$ Cardiothorac Surg 1995;9:330-4.

6 Aranki SF, Santini F, Adams DH, et al. Aortic valve endocarditis. Determinants of early survival and late morbidity. Circulation 1994;90(suppl II): 175-82.

7 Olaison L, Hogevik H, Myken P, et al. Early surgery in Olaison L, Hogevik H, Myken P, et al. Ear
infective endocarditis. $O \mp M$ 1996;89:267-78.

8 Reinhartz O, Herrmann M, Redling F, et al. Timing of surgery in patients with acute infective endocarditis. $\mathcal{F}$ Cardiovasc Surg 1996;37:397-400.

9 Choussat R, Thomas D, Isnard R, et al. Perivalvular abscesses associated with endocarditis. Clinical features and prognostic factors of overall survival in a series of 233 cases. Eur Heart f 1999;20:232-41.

10 Durack DT, Lukes AS, Bright DK. New criteria for diagnosis of infective endocarditis: utilization of specific echocardiographic findings. Am $\mathcal{F}$ Med 1994;96:200-9.

11 Bauernschmitt R, Vahl CF, Lange R, et al. Surgical treatment of acute endocarditis of the aortic valve with paravalvular abscess: considerations justifying the use of mechanical replacement devices. Eur $\mathcal{F}$ Cardiothorac Surg 1996;10:741-7.

12 Muehrcke DD, Cosgrove DM, Lytle BW, et al. Is there an advantage to repairing infected mitral valves? Ann Thorac Surg 1997;63:1718-24.

13 D'Agostino RS, Miller DC, Stinson EB, et al. Valve replacement in patients with native valve endocarditis: what really ment in patients with native valve endocarditis: what really determin 429 -38.

14 Aranki SF, Adams DH, Rizzo RJ, et al. Determinants of early mortality and late survival in mitral valve endocarditis. Circulation 1995;92(suppl II):143-9.

15 D'Udekem Y, David TE, Feindel CM, et al. Long-term results of operation for paravalvular abscess. Ann Thorac Surg 1996;62:48-53.

16 Watanabe G, Haverich A, Speier R, et al. Surgical treatment of active infective endocarditis with paravalvular involvement. F Thorac Cardiovasc Surg 1994;107:171-7.

17 Niwaya K, Knott-Craig CJ, Santangelo K, et al. Advantage of autograft and homograft valve replacement for complex aortic valve endocarditis. Ann Thorac Surg 1999;67:1603 8.

18 Grandmougin D, Prat A, Fayad G, et al. Acute aortic endocarditis with annular destruction: assessment of surgical treatment with cryopreserved valvular homografts. $\mathcal{F}$ Heart Valve Dis $1999 ; 8: 234-41$.

19 Pettersson G, Tingleff J, Joyce FS. Treatment of aortic valve endocarditis with the Ross operation. Eur $\mathcal{F}$ Cardiothorac Surg 1998;13:678-84.

20 Grover FL, Cohen DJ, Oprian C, et al. Determinants of the occurrence of and survival from prosthetic valve endocarditis. Experience of the Veterans Affairs Cooperative Study on valvular heart disease. I Thorac Cardiovasc Surg 1994;108:207-14

21 Mullany CJ, Chua YL, Schaff HV, et al. Early and late survival after surgical treatment of culture-positive active vival after surgical treatment of culture-posit

22 Haydock D, Barratt-Boyes B, Macedo T, et al. Aortic valve replacement for active infectious endocarditis in 108 patients. A comparison of freehand allograft valves with mechanical prostheses and bioprostheses. $\mathcal{F}$ Thorac Cardiovasc Surg 1992;103:130-9.

23 Larbalestier RI, Kinchla NM, Aranki SF, et al. Acute bacterial endocarditis. Optimizing surgical results. Circulation 1992;86 (suppl II):68-74.

24 Petrou M, Wong K, Albertucci M, et al. Evaluation of unstented aortic homografts for the treatment of prosthetic aortic valve endocarditis. Circulation 1994;90(part 2):198204.

25 Zwischenberger JB, Shalaby TZ, Conti VR. Viable cryopreserved aortic homograft for aortic valve endocarditis an annular abscesses. Ann Thorac Surg 1989;48:365-70.

26 Pagano D, Allen SM, Bonser RS. Homograft aortic valve and root replacement for severe destructive native or prosthetic endocarditis. Eur f Cardiothorac Surg 1994;8:173-6.

27 Dossche KM, Defauw JJ, Ernst SM, et al. Allograft aortic root replacement in prosthetic aortic valve endocarditis: a review of 32 patients. Ann Thorac Surg 1997;63:1644-9.

28 O'Brien MF, Stafford EG, Gardner MA, et al. A comparison of aortic valve replacement with viable cryopreserved and fresh allograft valves, with a note on chromosomal studies. F Thorac Cardiovasc Surg 1987;94:812-23.

29 McGiffin DC, Galbraith AJ, McLachlan GL, et al. Aortic valve infection. Risk factors for death and recurrent endocarditis after aortic valve replacement. $\mathcal{F}$ Thorac Cardiovasc Surg 1992;104:511-20.

30 Tornos MP, Olona M, Almirante B, et al. Infective endocarditis in the last 22 years. Why mortality remains high? [abstract]. Eur Heart f 1998;19(suppl):256.

31 Bauernschmitt R, Jakob HG, Vahl CF, et al. Operation for infective endocarditis: results after implantation of mechanical valves. Ann Thorac Surg 1998;65:359-64

32 Dearani JA, Orszulak TA, Schaff HV, et al. Results of allograft aortic valve replacement for complex endocarditis. allograft aortic valve replacement for complex

33 Edwards MB, Ratnatunga CP, Dore CJ, et al. Thirty-day mortality and long-term survival following surgery for prosthetic endocarditis: a study from the UK heart valve registry. Eur f Cardiothorac Surg 1998;14:156-64.

34 D'Udekem Y, David TE, Feindel CM, et al. Long-term results of surgery for active infective endocarditis. Eur 7 Cardiothorac Surg 1997;11:46-52.

35 Jault F, Gandjbakhch I, Rama A, et al. Active native valve endocarditis: determinants of operative death and late mortality. Ann Thorac Surg 1997;63:1737-41. 